\title{
ISVASE: identification of sequence variant associated with splicing event using RNA- seq data
}

\author{
Hasan Awad Aljohi ${ }^{1 \dagger}$, Wanfei Liu ${ }^{1,2,3+} \mathbb{D}$, Qiang Lin ${ }^{1,2+}$, Jun $\mathrm{Yu}^{1,2^{*}}$ and Songnian $\mathrm{Hu}^{1,2^{*}}$
}

\begin{abstract}
Background: Exon recognition and splicing precisely and efficiently by spliceosome is the key to generate mature mRNAs. About one third or a half of disease-related mutations affect RNA splicing. Software PVAAS has been developed to identify variants associated with aberrant splicing by directly using RNA-seq data. However, it bases on the assumption that annotated splicing site is normal splicing, which is not true in fact.

Results: We develop the ISVASE, a tool for specifically identifying sequence variants associated with splicing events (SVASE) by using RNA-seq data. Comparing with PVAAS, our tool has several advantages, such as multi-pass stringent ruledependent filters and statistical filters, only using split-reads, independent sequence variant identification in each part of splicing (junction), sequence variant detection for both of known and novel splicing event, additional exon-exon junction shift event detection if known splicing events provided, splicing signal evaluation, known DNA mutation and/or RNA editing data supported, higher precision and consistency, and short running time. Using a realistic RNA-seq dataset, we performed a case study to illustrate the functionality and effectiveness of our method. Moreover, the output of SVASES can be used for downstream analysis such as splicing regulatory element study and sequence variant functional analysis.
\end{abstract}

Conclusions: ISVASE is useful for researchers interested in sequence variants (DNA mutation and/or RNA editing) associated with splicing events. The package is freely available at https://sourceforge.net/projects/isvase/.

Keywords: Sequence variant, Splicing event, Association, RNA-seq, DNA mutation, RNA editing

\section{Background}

Alternative splicing is a normal phenomenon in eukaryotes and greatly increase the biodiversity of proteins. About $95 \%$ of multi-exonic genes are alternatively spliced in human [1]. The extreme example is the Drosophila Dscam gene, which produces thousands of protein isoforms by alternative splicing [2]. Classic pre-mRNA splicing is recognized and regulated by core splicing signals ( $5^{\prime}$ splice site ( $\left.5^{\prime} \mathrm{ss}\right), 3^{\prime}$ splice site ( $\left.3^{\prime} \mathrm{ss}\right)$, branch point sequence) and auxiliary sequences (splicing regulatory elements). Aberrant RNA splicing has become a common disease-causing mechanism, which can lead to hereditary disorders and cancers. Recent studies indicate that one

\footnotetext{
* Correspondence: junyu@big.ac.cn; husn@big.ac.cn

${ }^{\dagger}$ Equal contributors

${ }^{1}$ Joint Center for Genomics Research (JCGR), King Abdulaziz City for Science and Technology and Chinese Academy of Sciences, Prince Turki Road, Riyadh 11442, Saudi Arabia

Full list of author information is available at the end of the article
}

third or a half of disease-causing mutations can affect RNA splicing [3, 4]. Therefore, identification of sequence variant associated with splicing event (SVASE) becomes a meaningful procedure to illustrate the pathogenesis of diseases. Usually, sequence variant can result in aberrant splicing by disturbing regulatory element sequence or changing splice site [5]. For example, two sequence variants in splicing regulatory elements induce the aberrant splicing of BRCA2 exon 7 [6]. Moreover, RNA editing also can effect RNA splicing in transcriptome level [7].

Nowadays, RNA-seq has become a routine method for gene expression calling in multiple studies and can be also used to identify sequence variant and splicing event simultaneously $[8,9]$. However, there is only one bioinformatic tool (PVAAS) available for directly identifying genome-wide SVASE [10], which has some shortages, such as dependency on known splicing sites, only for novel splicing events, high false positive and long running time. Herein, we develop ISVASE, a suite of Perl 
scripts, to address the shortcomings of PVAAS and provide new functions for downstream analysis. The only necessary input files are genome sequence (FASTA format) and sequence alignment (BAM or SAM format) [11] files. The sequence alignment file must contain split-reads mapping result produced by software like GSNAP [12] and TopHat [13]. We also recommend users to provide known splicing events in GTF, GFF or BED format for junction shift event identification if concerned.

\section{Implementation}

The basic working principle of SVASE identification includes three main steps: (1) identify alternative splicing events; (2) identify sequence variants in specific splicing event using split-reads; and (3) evaluate the association between sequence variants and splicing events (see Fig. 1).

Based on sequence alignment result, ISVASE first filters mapped reads using stringent rule-dependent filters, such as low base quality $(<\mathrm{Q} 30)$, low mapping quality (unpaired reads for paired-end data, PCR duplication, quality control, multiple mapping, mismatch, insertion and deletion) and short read length $(<30 \mathrm{bp})$. Only splitreads with at least $8 \mathrm{bp}$ anchor size in both parts of splicing event (junction) can be used to identify putative splicing event. Initially, splicing events with low read depth $(<3)$ are discarded. Low abundant splicing events are also filtered out as background expression by applying Fisher's exact test to the putative splicing event and its related splicing events (sharing 5'ss or 3'ss). Here,
ISVASE divides each splicing event into two independent parts based on 5'ss and 3'ss. ISVASE can remove known splicing events using annotation file in GTF, GFF or BED format by option "-k no". Although excellent software for sequence variant calling has existed such as GATK [14] and samtools [15], their results are hard to be used for SVASE calling, which needs to clarify specific sequence variants for unique splicing event. Thus, ISVASE adopts de novo sequence variant identification by only using junction-supporting split-reads. The observed sequence variant candidates are filtered by following criteria: reads depth $(<3)$, alternative allele (ALT) supporting reads number $(<3)$, ALT proportion $(<0.1)$ and the significance of variant ( $p>0.05$, Fisher's exact test).

The practice of SVASE identification has a bit difference depending on whether the ALT frequencies are consistent between target splicing event and all related splicing events. We calculated the ALT frequencies for each sequence variants using reads of all splicing events and the target splicing event, respectively. If consistence, the association is assessed only using reads from target splicing event. Otherwise, total related reads are used. ISVASE applies same method as PVAAS to evaluate the significance of association. Besides, ISVASE assesses splicing signal by MaxEntScan [16] and identifies junction shift events to reduce the false positive of splicing event calling. Furthermore, DNA mutation and/or RNA editing profiles (like dbSNP [17], DARNED [18], RADAR [19] or user provided DNA mutation or RNA editing

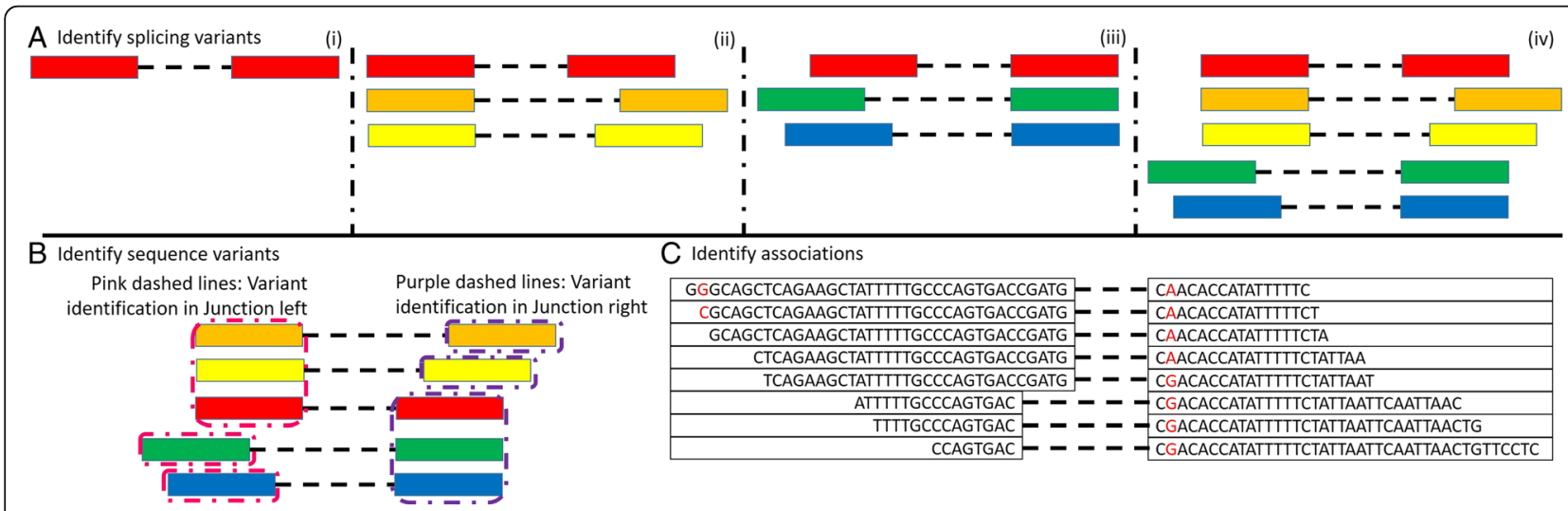

Fig. 1 Schematic diagram of the ISVASE software. a Identify splicing variants in RNA-seq data. All splicing variants can be divided into four types according to relationship between target splicing variant (red colour) and other splicing variants (from left to right): (i) unique splicing variant; (ii) splicing variants with same junction start; (iii) splicing variants with same junction end; and (iv) splicing variants with same junction start or end. b Identify sequence variants for each splicing variant and all related splicing variants. To handle all splicing variant types, we identify sequence variants for two parts of splicing separately. In the left part, for junctions with orange, yellow and red colour, the all related splicing variants should be three (all these junctions); however, for junctions with green and blue colour, the total junction is one (itself). Similarly, in the right part, junctions with red, green and blue colour have three all related splicing variants while junctions with orange and yellow colour only has one related junction (itself). c Identify associations. This step includes three significant judgements for sequence variants, junction existence and association between sequence variants and junctions, respectively. The example shown two junctions with same junction end. For junction one (top), two sequence variants are identified (left $\mathrm{G}($ ref)- $>C($ alt $)$ and right $\mathrm{G}($ ref)- > A(alt)). In sequence variant significant judgement, left is filtered ( $p$ value $=1$ ) while right passes the test ( $p$ value $=0.0476$ ). In junction significant judgement and association judgement, $p$ value of top junction is 0.0128 (significant) and 0.0070 (significant) respectively. Dashed lines represent gaps in the alignment 
Table 1 The statistics of SVASE identification using PVAAS and ISVASE

\begin{tabular}{|c|c|c|c|c|c|c|c|c|c|}
\hline \multirow[t]{2}{*}{ Data } & \multicolumn{3}{|c|}{ PVAAS } & \multicolumn{3}{|c|}{ ISVASE(novel) } & \multicolumn{3}{|c|}{ ISVASE(all) } \\
\hline & Total & $\mathrm{dbSNP}$ & RADAR & Total & $\mathrm{dbSNP}$ & RADAR & Total & $\mathrm{dbSNP}$ & RADAR \\
\hline PVAAS test data & 8 & 0 & 0 & 14 & 7 & 0 & 172 & 129 & 0 \\
\hline Control1 (SRR388226) & 61 & 12 & 0 & 134 & 54 & 1 & 2577 & 2138 & 3 \\
\hline Control2(SRR388227) & 63 & 9 & 0 & 120 & 50 & 2 & 2557 & 2130 & 3 \\
\hline Control(common) & 28 & 2 & 0 & 87 & 36 & 1 & 2105 & 1788 & 2 \\
\hline Knockdown1(SRR388228) & 93 & 18 & 0 & 187 & 83 & 1 & 2710 & 2250 & 2 \\
\hline Knockdown2(SRR388229) & 89 & 24 & 0 & 168 & 73 & 1 & 2760 & 2293 & 1 \\
\hline Knockdown(common) & 31 & 8 & 0 & 119 & 55 & 1 & 2298 & 1951 & 1 \\
\hline
\end{tabular}

sites) can be used to assign the source type of sequence variants. ISVASE outputs the detailed statistical results with figures and tables. ISVASE also extracts the flanking sequence for sequence variants, which can be used to predict exonic splicing enhancer (ESE) motifs using tools like ESEfinder [20] and Human Splicing Finder [21]. The output of identified SVASEs can be accepted by ANNOVAR [22] and SnpEff [23] for further functional analysis like KEGG pathway and Gene Ontology. The code of ISVASE was written using Perl (v5.18.4), the figures were created by $\mathrm{R}$ (v3.1.2) while the sequence alignment file was operated by samtools (v1.2).

\section{Results and Discussion}

To demonstrate the functionality of ISVASE and compare with PVAAS, PVAAS testing data (downloaded from website http://pvaas.sourceforge.net/) was used. PVAAS (v0.1.5) identified 8 SVASEs (belonging to new splicing events), while ISVASE obtained 172 SVASEs and 14 of them were new splicing events (Table 1, Additional files 1 and 2). Two software only share one SVASE, which probably is genuine according to dbscSNV [24]. Among other 7 PVAAS unique SVASEs, 1 SVASE has a low ALT ratio $(<=0.01), 1$ SVASE is supported by un-split reads and remaining 5 SVASEs are identified only by a small part of

Table 2 The performance comparison between PVAAS and ISVASE

\begin{tabular}{|c|c|c|c|}
\hline Data & Method & Precision & Consistency \\
\hline \multirow[t]{3}{*}{ PVAAS test data } & PVAAS & $0.00(0 / 8)$ & - \\
\hline & ISVASE(novel) & $0.50(7 / 14)$ & - \\
\hline & ISVASE(all) & $0.75(129 / 172)$ & - \\
\hline \multirow[t]{3}{*}{ Control1(SRR388226) } & PVAAS & $0.20(12 / 61)$ & $0.46(28 / 61)$ \\
\hline & ISVASE(novel) & $0.40(54 / 134)$ & $0.65(87 / 134)$ \\
\hline & ISVASE(all) & $0.83(2138 / 2577)$ & $0.82(2105 / 2577)$ \\
\hline \multirow[t]{4}{*}{ Control1 (SRR388227) } & PVAAS & $0.14(9 / 63)$ & $0.44(28 / 63)$ \\
\hline & ISVASE(novel) & $0.42(50 / 120)$ & $0.73(87 / 120)$ \\
\hline & ISVASE(all) & $0.83(2130 / 2557)$ & $0.82(2105 / 2557)$ \\
\hline & PVAAS & $0.07(2 / 28)$ & - \\
\hline \multirow[t]{2}{*}{ Control(common) } & ISVASE(novel) & $0.41(36 / 87)$ & - \\
\hline & ISVASE(all) & $0.85(1788 / 2105)$ & - \\
\hline \multirow[t]{3}{*}{ Knockdown1(SRR388228) } & PVAAS & $0.19(18 / 93)$ & $0.33(31 / 93)$ \\
\hline & ISVASE(novel) & $0.44(83 / 187)$ & $0.64(119 / 187)$ \\
\hline & ISVASE(all) & $0.83(2250 / 2710)$ & $0.85(2298 / 2710)$ \\
\hline \multirow[t]{3}{*}{ Knockdown2(SRR388229) } & PVAAS & $0.27(24 / 89)$ & $0.35(31 / 89)$ \\
\hline & ISVASE(novel) & $0.43(73 / 168)$ & $0.71(119 / 168)$ \\
\hline & ISVASE(all) & $0.83(2293 / 2760)$ & $0.83(2298 / 2760)$ \\
\hline \multirow[t]{3}{*}{ Knockdown(common) } & PVAAS & $0.26(8 / 31)$ & - \\
\hline & ISVASE(novel) & $0.46(55 / 119)$ & - \\
\hline & ISVASE(all) & $0.85(1951 / 2298)$ & - \\
\hline
\end{tabular}


Table 3 The running time comparison between PVAAS and ISVASE

\begin{tabular}{llll}
\hline Data & PVAAS & ISVASE(novel) & ISVASE (all) \\
\hline PVAAS test data & 1h38m25s & $11 \mathrm{~m} 22 \mathrm{~s}$ & $13 \mathrm{~m} 11 \mathrm{~s}$ \\
Control1(SRR388226) & 12h5m22s & 2h27m31s & 2h52m33s \\
Control2(SRR388227) & $12 \mathrm{~h} 52 \mathrm{~m} 19 \mathrm{~s}$ & $2 \mathrm{~h} 29 \mathrm{~m} 50 \mathrm{~s}$ & $2 \mathrm{~h} 53 \mathrm{~m} 17 \mathrm{~s}$ \\
Knockdown1(SRR388228) & $15 \mathrm{~h} 45 \mathrm{~m} 40 \mathrm{~s}$ & $2 \mathrm{~h} 37 \mathrm{~m} 36 \mathrm{~s}$ & $3 \mathrm{~h} 4 \mathrm{~m} 3 \mathrm{~s}$ \\
Knockdown2(SRR388229) & 16h40m40s & 2h42m27s & $3 \mathrm{~h} 9 \mathrm{~m} 38 \mathrm{~s}$ \\
\hline
\end{tabular}

target junction supporting reads $(0.8 \% \sim 8 \%)$. All of these error-prone SVASEs have been filtered in our tool. All 14 SVASEs belonging to new splicing events in our result have high confident evidences such as mapping quality, ALT reads and other filter criteria mentioned above. Among 158 SVASEs in known splicing events, 55 SVASEs are non-reference homozygous, 66 SVASEs have more than $80 \%$ ALT reads, and 110 SVASEs have more than $50 \%$ ALT reads. Comparing SVASEs with dbSNP and RADAR database, we found that ISVASE has better performance than PVAAS both for novel and all SVASEs (Table 2). Moreover, ISVASE run faster than PVAAS. For test data (7.26 million reads), PVAAS takes $1.63 \mathrm{~h}$, while ISVASE only needs $11 \mathrm{~min}$ for novel splicing events or 13 min for all splicing events (Table 3).

To further reveal the advantage of ISVASE, we also test another real data set with 4 RNA-seq samples for human glioblastoma cell line U87MG (SRR388226 and SRR388227 are control samples and SRR388228 and SRR388229 are ADAR knockdown samples) [25]. The raw data was trimmed by Trimmomatic [26] and aligned by GSNAP (only concordant mapping results were used for downstream analysis) [12]. Using ISVASE, 134 and 120 SVASEs (87 common) were obtained for control data, while 187 and 168 SVASEs (119 common) for knockdown data in new splicing events. If considering all splicing events, 2105 and 2298 common SVASEs were identified in control and knockdown data (Table 1, Additional files $3,4,5$, and 6). In each sample, at most three SVASEs belonging to RNA editing sites in RADAR database were detected (totally four SVASEs belonging to RADAR database), and more than $82 \%$ SVASEs existed in dbSNP. In comparison, PVAAS got 61 and 63 SVASEs (28 common) for control data, while 93 and 89 SVASEs (31 common) for knockdown data (Table 1, Additional files 7, 8, 9, and 10). In PVAAS result, there wasn't any SVASE belonging to RNA editing sites in RADAR database and at most 27\% SVASEs existed in dbSNP. These results indicated that PVAAS has higher false positive rate comparing with ISVASE (Table 2). Using repeat samples, we also found that PVAAS has lower consistency rate comparing with ISVASE (about 47\% vs. about 83\%) (Table 2). Moreover, for each sample, ISVASE showed an advantage of running time to PVAAS (about $3 \mathrm{~h}$ vs. 14.34 h) (Table 3).

The SVASEs identified by ISVASE can be used for downstream analysis easily. For example, we used 65 common SVASEs in new splicing events from the above four samples to do further analysis. We annotated these SVASEs by ANNOVAR and found 28 related genes (Additional file 11). Among them, 20, 9 and 8 SVASEs located in $H L A, H C G 4 B$ and AHNAK2 genes. HLA genes play important roles in tumor immune surveillance and escape, and $H C G 4 B$ gene is a pseudogene of HLA complex group. AHNAK2 gene is associated with

Table 4 Gene Ontology enrichment analysis for genes related with 65 common SVASEs using PANTHER (filtered redundant records)

\begin{tabular}{|c|c|c|c|c|c|}
\hline GO function & Total gene & SVASE gene & Expected & Fold Enrichment & $P$ value $(<0.05)$ \\
\hline \multicolumn{6}{|l|}{ GO biological process complete } \\
\hline $\begin{array}{l}\text { antigen processing and presentation of endogenous } \\
\text { peptide antigen via MHC class I }\end{array}$ & 15 & 3 & 0.02 & $>100$ & 0.00541 \\
\hline $\begin{array}{l}\text { antigen processing and presentation of peptide } \\
\text { antigen via MHC class I }\end{array}$ & 108 & 6 & 0.12 & 50.28 & $1.51 \mathrm{E}-05$ \\
\hline $\begin{array}{l}\text { antigen processing and presentation of endogenous } \\
\text { antigen }\end{array}$ & 19 & 3 & 0.02 & $>100$ & 0.011 \\
\hline $\begin{array}{l}\text { antigen processing and presentation of exogenous } \\
\text { antigen }\end{array}$ & 181 & 6 & 0.2 & 30 & 0.000317 \\
\hline response to type I interferon & 74 & 6 & 0.08 & 73.37 & $1.6 \mathrm{E}-06$ \\
\hline response to interferon-gamma & 151 & 6 & 0.17 & 35.96 & 0.000109 \\
\hline \multicolumn{6}{|l|}{ GO molecular function complete } \\
\hline antigen binding & 107 & 6 & 0.12 & 50.75 & 4.69E-06 \\
\hline \multicolumn{6}{|l|}{ GO cellular component complete } \\
\hline MHC protein complex & 30 & 6 & 0.03 & $>100$ & $1.14 \mathrm{E}-09$ \\
\hline membrane-bounded vesicle & 1169 & 9 & 1.29 & 6.97 & 0.00285 \\
\hline vesicle membrane & 508 & 7 & 0.56 & 12.47 & 0.00116 \\
\hline
\end{tabular}


calcium channel proteins and its exon 7 size is almost $18 \mathrm{~kb}$. We found 8 SVASEs associated with 5 new splicing events inside the exon 7. Gene Ontology enrichment analysis found these 28 genes are significantly enriched in cancer related functions, such as antigen processing and presentation, response to type I interferon and interferon-gamma (Table 4). We also used ESEfinder to detect ESE motifs and found 57 of 65 SVASEs located in predicted ESE motifs. This result indicates most of SVASEs perform their function possibly by influencing ESE motifs of splicing events. Moreover, SVASEs have some basic characteristics (using SRR388226 data as an example), such as high proportion of canonical splicing signal GT-AG (or reverse complement $\mathrm{CT}-\mathrm{AC}$ ), similar signal scores for splice

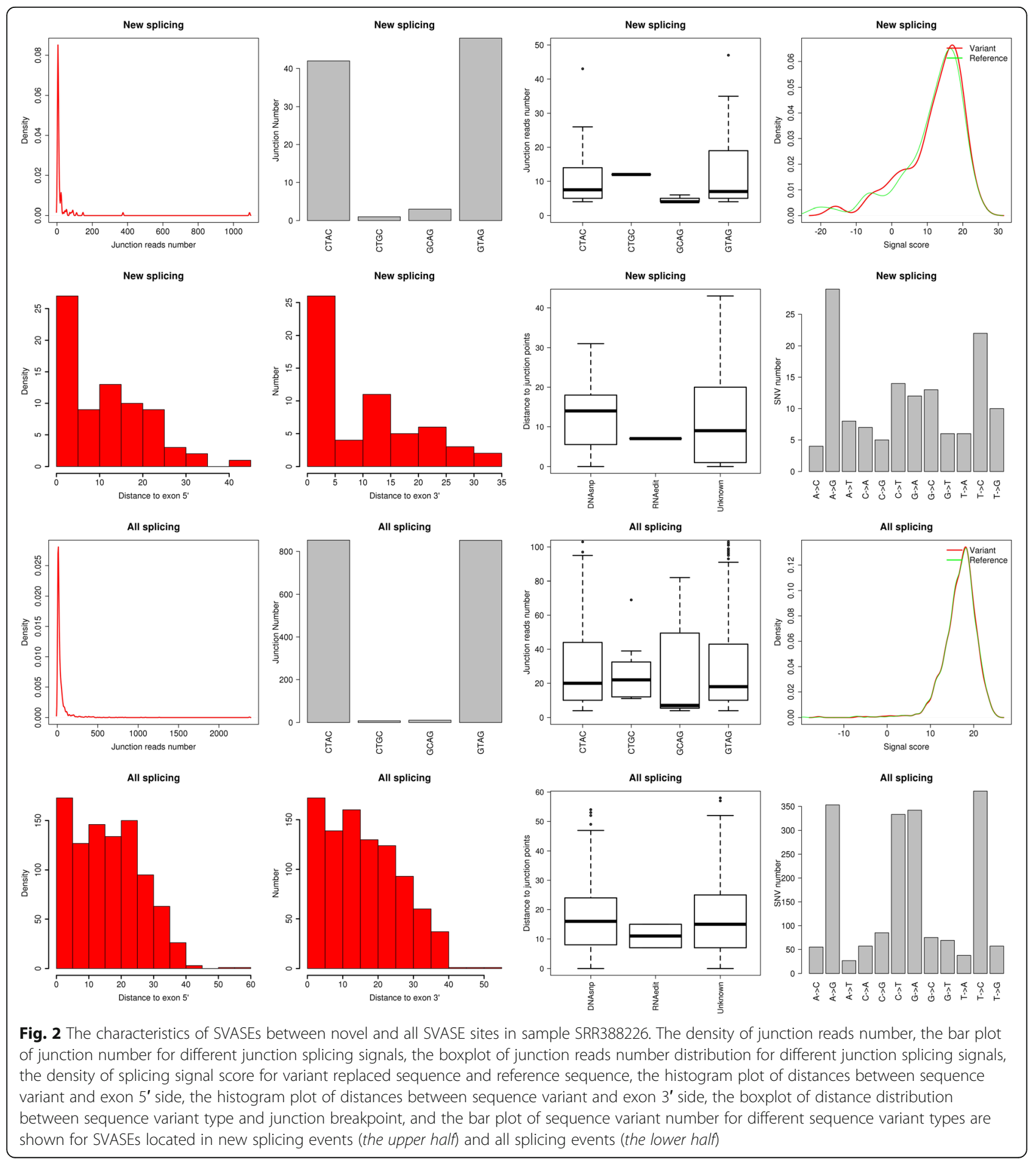


sites with reference or alternative allele, tendency to junction breakpoints, and high frequency of A- > G/T- > C and G- > A/C- > T transitions (58.96\% in new splicing events and $75.13 \%$ in all splicing events) (Fig. 2).

\section{Conclusions}

ISVASE provides users to identify SVASEs simply and fast using RNA-seq data. It identifies SVASEs for both parts of splicing event (or junction) separately. To reduce false positives due to sequencing errors, ISVASE applies several stringent rule-depended filters and statistical filters in different steps. ISVASE can evaluate junction shift events and junction signals ( $5^{\prime}$ ss and $3^{\prime}$ ss) to remove false positive splicing events. It also can use user provided DNA mutation and/or RNA editing data to designate types of sequence variants. To facilitate downstream analysis, ISVASE obtains flanking sequences and VCF output for other tools usage. ISVASE also provides 6 tables and 8 figures to describe the characteristics of SVASEs. In summary, our approach enabled de novo identification of SVASEs, which sets the stage for further mechanistic studies.

\section{Additional files}

Additional file 1: PVAAS result for its test data. (XLS 753 bytes)
Additional file 2: ISVASE result for PVAAS test data. (XLS $17 \mathrm{~kb})$
Additional file 3: ISVASE result for SRR388226. (XLS $365 \mathrm{~kb})$
Additional file 4: ISVASE result for SRR388227. (XLS $362 \mathrm{~kb})$
Additional file 5: ISVASE result for SRR388228. (XLS $384 \mathrm{~kb})$
Additional file 6: ISVASE result for SRR388229. (XLS $391 \mathrm{~kb})$
Additional file 7: PVAAS result for SRR388226. (XLS $4 \mathrm{~kb})$
Additional file 8: PVAAS result for SRR388227. (XLS $4 \mathrm{~kb})$
Additional file 9: PVAAS result for SRR388228. (XLS $6 \mathrm{~kb})$
Additional file 10: PVAAS result for SRR388229. (XLS $6 \mathrm{~kb})$
Additional file 11: Genes of 65 common SVASEs in new splicing events
identified by ISVASE for four samples. (DOCX 12 kb)

\section{Abbreviations}

3' ss: 3' splice site; 5' ss: 5' splice site; ALT: alternative allele; ESE: exonic splicing enhancer; ISVASE: Identification of sequence variant associated with splicing event; PVAAS: Program to identify variants associated with aberrant splicing; SE: splicing event; SV: Sequence variant; SVASE: Sequence variant associated with splicing event

\section{Acknowledgements}

Technical supports were provided by the CAS Key Laboratory of Genome Science and Information, Beijing Institute of Genomics, Chinese Academy of Sciences, the People's Republic of China. The authors thank the anonymous reviewers for critical comments and helpful suggestions.

\section{Funding}

This study is supported by grants from National Natural Science Foundation of China (Grant No. 31501042, 31,271,385 and 31,200,957), the Strategic Priority Research Program of the Chinese Academy of Sciences (Grant No. XDA08020102), and KACST grant 1035-35 from King Abdulaziz City for Science and Technology (KACST), Kingdom of Saudi Arabia. None of the funding bodies have played any part in the design of the study, in the collection, analysis, and interpretation of the data, or in the writing of the manuscript.

\section{Availability of data and materials}

ISVASE package is freely available at https://sourceforge.net/projects/isvase/ All data generated or analyzed during this study are included in this article and its supplementary information files.

Project name: ISVASE

Operating system: Unix/Linux

Programming language: Perl

Other requirements: Perl Environment (perl v5.18.4 or later), Perl module Text::NSP and Statistics::Multtest, R Environment (R 3.1.2 or later), samtools (v1.2)

License: GNU General Public License version 3.0 (GPLv3)

Any restrictions to use by non-academics: None

\section{Author's contributions}

HAA, WFL and QL contributed equally to this work. HAA, WFL and QL written the codes for tool. HAA, WFL, QL, SNH and JY led the research and wrote the manuscript. All authors read and approved the final manuscript.

\section{Competing interests}

The authors declare that they have no competing interests.

\section{Consent for publication}

Not applicable.

Ethics approval and consent to participate Not applicable.

\section{Publisher's Note}

Springer Nature remains neutral with regard to jurisdictional claims in published maps and institutional affiliations.

\section{Author details}

${ }^{1}$ Joint Center for Genomics Research (JCGR), King Abdulaziz City for Science and Technology and Chinese Academy of Sciences, Prince Turki Road, Riyadh 11442, Saudi Arabia. ${ }^{2}$ CAS Key Laboratory of Genome Sciences and Information, Beijing Institute of Genomics, Chinese Academy of Sciences, NO.1 Beichen West Road, Chaoyang District, Beijing 100101, China. ${ }^{3}$ Current address: Grail Scientific Co. Ltd., Room 26-1, Build A, Meilong Jiayuan, NO. 80 South Nanjing Street, Heping District, Shenyang, Liaoning 110000, China.

Received: 21 December 2016 Accepted: 15 June 2017

Published online: 28 June 2017

\section{References}

1. Pan Q, Shai O, Lee LJ, Frey BJ, Blencowe BJ. Deep surveying of alternative splicing complexity in the human transcriptome by high-throughput sequencing. Nat Genet. 2008;40(12):1413-5.

2. Sun W, You X, Gogol-Döring A, He H, Kise Y, Sohn M, et al. Ultra-deep profiling of alternatively spliced Drosophila Dscam isoforms by circularization-assisted multi-segment sequencing. EMBO J. 2013;32(14): 2029-38.

3. Lim KH, Ferraris L, Filloux ME, Raphael BJ, Fairbrother WG. Using positional distribution to identify splicing elements and predict pre-mRNA processing defects in human genes. Proc Natl Acad Sci. 2011;108(27):11093-8.

4. Cartegni $\mathrm{L}$, Chew SL, Krainer AR. Listening to silence and understanding nonsense: exonic mutations that affect splicing. Nat Rev Genet. 2002;3(4): 285-98.

5. Ward AJ, Cooper TA. The pathobiology of splicing. J Pathol. 2010;220(2): 152-63.

6. Gaildrat P, Krieger S, Di Giacomo D, Abdat J, Révillion F, Caputo S, et al. Multiple sequence variants of BRCA2 exon 7 alter splicing regulation. J Med Genet. 2012:49(10):609-17.

7. Schoft VK, Schopoff S, Jantsch MF. Regulation of glutamate receptor B premRNA splicing by RNA editing. Nucleic Acids Res. 2007;35(11):3723-32.

8. Adamopoulos PG, Kontos CK, Tsiakanikas P, Scorilas A. Identification of novel alternative splice variants of the BCL2L12 gene in human cancer cells, using next-generation sequencing methodology. Cancer Lett. 2016; 
9. Li YI, van de Geijn B, Raj A, Knowles DA, Petti AA, Golan D, et al. RNA splicing is a primary link between genetic variation and disease. Science. 2016;352(6285):600-4.

10. Wang L, Nie JJ, Kocher J-PA. PVAAS: identify variants associated with aberrant splicing from RNA-seq. Bioinformatics. 2015;31(10):1668-70.

11. Li H, Handsaker B, Wysoker A, Fennell T, Ruan J, Homer N, et al. The sequence alignment/map format and SAMtools. Bioinformatics. 2009;25(16):2078-9.

12. Wu TD, Nacu S. Fast and SNP-tolerant detection of complex variants and splicing in short reads. Bioinformatics. 2010;26(7):873-81.

13. Trapnell C, Pachter L, Salzberg SL. TopHat: discovering splice junctions with RNA-Seq. Bioinformatics. 2009;25(9):1105-11.

14. McKenna A, Hanna M, Banks E, Sivachenko A, Cibulskis K, Kernytsky A, et al. The Genome Analysis Toolkit: a MapReduce framework for analyzing nextgeneration DNA sequencing data. Genome Res. 2010;20(9):1297-303.

15. Li H. A statistical framework for SNP calling, mutation discovery, association mapping and population genetical parameter estimation from sequencing data. Bioinformatics. 2011;27(21):2987-93.

16. Yeo G, Burge CB. Maximum entropy modeling of short sequence motifs with applications to RNA splicing signals. J Comput Biol. 2004;11(2-3):377-94.

17. Sherry ST, Ward M-H, Kholodov M, Baker J, Phan L, Smigielski EM, et al. dbSNP: the NCBI database of genetic variation. Nucleic Acids Res. 2001; 29(1):308-11.

18. Kiran A, Baranov PV. DARNED: a DAtabase of RNa EDiting in humans. Bioinformatics. 2010;26(14):1772-6.

19. Ramaswami G, Li JB: RADAR: a rigorously annotated database of A-to-I RNA editing. Nucleic Acids Res. 2013. doi:10.1093/nar/gkt99.

20. Cartegni L, Wang J, Zhu Z, Zhang MQ, Krainer AR. ESEfinder: a web resource to identify exonic splicing enhancers. Nucleic Acids Res. 2003;31(13):3568-71.

21. Desmet F-O, Hamroun D, Lalande M, Collod-Béroud G, Claustres M, Béroud C. Human Splicing Finder: an online bioinformatics tool to predict splicing signals. Nucleic Acids Res. 2009;37(9):e67.

22. Wang K, Li M, Hakonarson H. ANNOVAR: functional annotation of genetic variants from high-throughput sequencing data. Nucleic Acids Res. 2010; 38(16):e164

23. Cingolani P, Platts A, Wang LL, Coon M, Nguyen T, Wang L, et al. A program for annotating and predicting the effects of single nucleotide polymorphisms, SnpEff: SNPs in the genome of Drosophila melanogaster strain w1118; iso-2; iso-3. Fly. 2012;6(2):80-92.

24. Jian X, Boerwinkle E, Liu X. In silico prediction of splice-altering single nucleotide variants in the human genome. Nucleic Acids Res. 2014;42(22): 13534-44.

25. Bahn JH, Lee J-H, Li G, Greer C, Peng G, Xiao X. Accurate identification of Ato-I RNA editing in human by transcriptome sequencing. Genome Res. 2012;22(1):142-50.

26. Bolger AM, Lohse M, Usadel B: Trimmomatic: a flexible trimmer for Illumina sequence data. Bioinformatics. 2014. doi:10.1093/bioinformatics/btu170.

\section{Submit your next manuscript to BioMed Central and we will help you at every step:}

- We accept pre-submission inquiries

- Our selector tool helps you to find the most relevant journal

- We provide round the clock customer support

- Convenient online submission

- Thorough peer review

- Inclusion in PubMed and all major indexing services

- Maximum visibility for your research

Submit your manuscript at www.biomedcentral.com/submit

) Biomed Central 